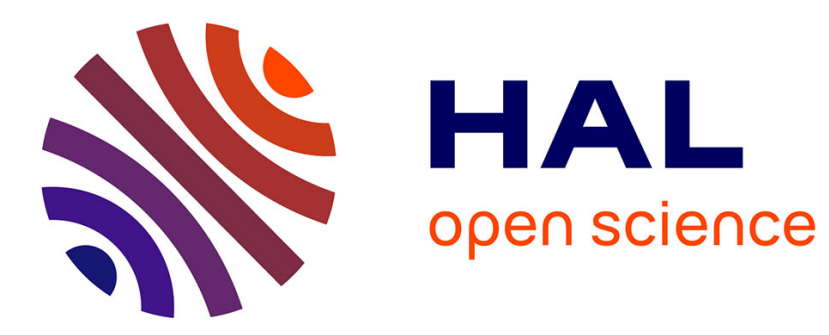

\title{
Detecting and estimating density dependence in wildlife populations
}

Jean-Dominique Lebreton, Olivier Gimenez

\section{To cite this version:}

Jean-Dominique Lebreton, Olivier Gimenez. Detecting and estimating density dependence in wildlife populations. Journal of Wildlife Management, 2012, 77, pp.12 - 23. 10.1002/jwmg.425 . hal03499389

\section{HAL Id: hal-03499389 \\ https://hal.science/hal-03499389}

Submitted on 25 Dec 2021

HAL is a multi-disciplinary open access archive for the deposit and dissemination of scientific research documents, whether they are published or not. The documents may come from teaching and research institutions in France or abroad, or from public or private research centers.
L'archive ouverte pluridisciplinaire HAL, est destinée au dépôt et à la diffusion de documents scientifiques de niveau recherche, publiés ou non, émanant des établissements d'enseignement et de recherche français ou étrangers, des laboratoires publics ou privés. 
DETECTING AND ESTIMATING DENSITY DEPENDENCE IN WILDLIFE

POPULATIONS

Jean-Dominique Lebreton, CEFE UMR 5175, CNRS, 1919 Route de Mende, 34293

Montpellier cedex 5, France

Olivier Gimenez, CEFE UMR 5175, CNRS, 1919 Route de Mende, 34293 Montpellier cedex 5, France

ABSTRACT We review methods for detecting and assessing the strength of density dependence (DD) based on two types of approaches: surveys of population size and studies of life history traits, in particular demographic parameters.

For the first type of studies, methods neglecting uncertainty in population size should definitely be abandoned. Bayesian approaches to simple state-space models accounting for uncertainty in population size are recommended, with some caution because of numerical difficulties and risks of model misspecification. Realistic state-space models incorporating features such as environmental covariates, age-structure, etc., may lack power because of the shortness of the time series and the simultaneous presence of process and sampling variability. In all cases, complementing the population survey data with some external information, in priority on the intrinsic growth rate, is highly recommended.

Methods for detecting DD in life history traits are generally conservative, i.e. tend to underestimate the strength of DD. Among approaches to correct for this effect, the state-space formulation of capture-recapture models is again the most promising.

Foreseeable developments will exploit integrated monitoring combining population size surveys and individual longitudinal data in refined state-space models, for which a Bayesian approach is the most straightforward statistical treatment. One may thus expect an integration 
of various types of models that will make it possible to look at DD as a complex biological process interacting with other processes rather than in terms of a simple equation: modern statistical and modeling tools make such a synthesis within reach.

KEYWORDS Animal demography, Bayesian methods, Density-dependence, Kalman filter, Population dynamics, State-space models, Wildlife

\section{INTRODUCTION}

The i-state philosophy (Diekmann 1985) describes demography at the individual level based on life-cycle stages. This philosophy naturally leads to describe death, fecundity, and more generally any transition between stages such as recruitment or dispersal, through per capita parameters. If such individual parameters are constant or at least stationary (in the sense of stochastic processes: affected by random effects that can be shifted over time, and, as such, in particular, not affected by a trend), the population growth is exponential, generally after damping of the effect of initial population structure, as well known for instance for matrix models (Caswell 2001) and their stochastic generalizations (Tuljapurkar, 1990). The key paradigm of exponential growth in population dynamics (Turchin 1981) is thus intimately linked to the i-state philosophy.

It has been recognized for more than two centuries (Malthus 1798) that exponential growth is impossible in the long term (Turchin 1981), as exponential growth leads to large population sizes that unavoidably induce depletion of resources and in turn of individual performance. This is a broad definition of Density-Dependence (DD hereafter), as a phenomenon that explicitly has to do with resource and individual performance. In passing, as most authors in this context, we loosely use the word density for population size or local population size, e.g. in the term DD itself. 
Density-Dependence became a key subject in population dynamics with the well known logistic growth curve (Verhulst 1838). One of the discrete time counterparts is the discrete time Gompertz model $N_{t+1}=\lambda N_{t}^{-b} N_{t}$ with $b>0$. Such models are phenomenological, as they describe only the population level and do not explicitly consider the individual level, contrary to mechanistic models such as, e.g., matrix models that translate individual parameters into population level consequences. There has been a continuous tension since the $\mathrm{XIX}^{\text {th }}$ century between phenomenological and mechanistic approaches to density-dependence (see Krebs 1995, Murdoch 1994) and continuing discussions (e.g. Berryman 2004) on the role of limitation by resource availability - an individual level phenomenon - as supposedly opposed to regulation by $\mathrm{DD}$, a population level result.

A key question is whether DD is present in a population, and if so, how to measure its intensity. As usual, even if one relies on some statistical significance for detection, one has indeed in mind some biological significance, i.e. the idea that DD is or is not negligible for the future fate of the population or relative to other sources of variation, and thus bears a relationship to its management (Guthery 2012). Indeed the measurement phase is often linked with the idea that population projections accounting for DD go one step beyond those based on constant parameters: there is still a strong assumption of stationarity in the projection but rather than "as if parameters were the same as up to now", it is "as if parameters were varying with density as they have been shown to do up to now". Estimating the strength of DD is also central in discussions of the relative role and magnitude of density-dependent and density-independent variation in population size, the subject of a famous controversy in the 50s (Andrewartha and Birch 1954, Lack 1954).

Not surprisingly, the tension between phenomenological / population and mechanistic / individual approaches has been pervasive in the issue of detection / estimation of DD, would 
it be only because the data themselves can be at the population level only (e.g. population surveys) or at the level of individual traits only (e.g. body weight data). Finally, statistical difficulties arise as the arrow of time induces dependencies that cannot be handled by naive statistical approaches. While a comprehensive review of the literature would be beyond reach, it is clear that the issue of detection / measurement of DD has alternatively raised optimistic and pessimistic points of view, and has been a subject of "frustration" (Dennis and Taper 1994). Can we take series of data and estimate in a simple fashion how many show densitydependence, as done by Brook and Bradshaw (2006) for 1198 species, or should we conclude with Krebs (1995) that DD is an unattainable holy grail?

The purpose of this paper is to attempt to review the subject of detection and estimation of DD with specific reference to these difficulties and the resulting confusing state of the art. Although we will use many equations, as "if you are faced by a difficulty or a controversy in science, an ounce of algebra is worth a ton of verbal argument" (a conviction of J.B.S.Haldane, according to Maynard-Smith 1965), this review will attempt to avoid technical developments (for a more technical review, see Lebreton 2009).

We will first review the methods for detection and estimation of DD based on population size estimates, in three steps: a) an attempt to formalize the role of resource and density, b) methods in the absence of uncertainty in population size, c) methods accounting for uncertainty in population size, a key feature for wild animal populations. Then we will review methods based on analyses of individual traits, demographic or not. We will then discuss new modeling opportunities and perspectives.

Calculations were done using our own Matlab ${ }^{\circledR}$ code, carefully validated by a series of crosschecks, and WinBUGS (Lunn et al. 2000). The Matlab ® code is made available as supplementary material. 

DETECTING AND ESTIMATING DENSITY DEPENDENCE BASED ON POPULATION SIZE ESTIMATES

The Gompertz model $N_{t+1}=\lambda N_{t}^{-b} N_{t}$ becomes linear on a log scale. Denoting $x_{t}=\operatorname{Ln}\left(N_{t}\right)$ and $r=\operatorname{Ln}(\lambda)$ :

$$
x_{t+1}=r+(1-b) x_{t}
$$

As recalled by Lebreton (2009), $r=\operatorname{Ln}(\lambda)$ is the growth rate for $N=1$, while the growth rate for $N=0$ is infinite. This bears no consequences on our treatment of this model and related ones, and we will speak of $r$ as the intrinsic growth rate.

Under $b=0$, i.e. density independence, the model reduces to

$$
x_{t+1}=r+x_{t}
$$

As the world is not deterministic, a straightforward stochastic generalization considers some extra random variation to represent demographic and environmental stochasticity, leading under density-independence and density-dependence, respectively, to:

$$
x_{t+1}=r+(1-b) x_{t}+\varepsilon_{t}
$$

$$
x_{t+1}=r+x_{t}+\varepsilon_{t}
$$

The random terms, represented by Greek letters, have expectations equal to 0 and, unless otherwise stated, are assumed to be normally distributed. The latter model is a random walk as, besides the shift $r, x_{t}$ varies through independent additive increments. Because of their simplicity, these models have been used by a number of authors over the years (see Dennis et al. 2006, Lebreton 2009). One can equivalently test for DD either by a test of model [3] vs model [4], such as a likelihood ratio test, or by a test of $\mathrm{H}_{0} b=0$ in model [3], e.g. as a Wald 
test. The latter approach is usually preferred as it can easily be easily implemented as a onetailed test of $\mathrm{H}_{0} b=0$ vs $\mathrm{H}_{1} b>0$, the alternative of biological interest.

\section{Density-dependence and resource-dependence}

By rewriting [3] one can make a per capita growth rate $r-b x_{t}+\varepsilon_{t}$ appear:

$$
x_{t+1}=\left(r-b x_{t}+\varepsilon_{t}\right)+x_{t}
$$

As DD occurs through the depletion of resource of some kind, in the per capita growth rate, the $\log$ population size $x_{t}$ is a proxy for something else. Let's assume a single latent variable $l_{t}$, such as the amount of a key resource left, is the actual determinant of the growth rate.

Assuming $l_{t}$ can be expressed on the same scale as $x_{t}$, the actual growth rate is then $r-b l_{t}+\varepsilon_{t}$. The $\log$-population size $x_{t}$ is then a proxy for $l_{t}$, with a relationship necessarily modified by some random variation, $l_{t}=x_{t}+\varsigma_{t}$, as the proxy and the latent variable cannot be expected to be perfectly correlated over time. The random term $\varsigma_{t}$ has a null expectation, i.e. $\left(E\left(\varsigma_{t}\right)=0\right.$. Note that this issue has nothing to do with uncertainty on $x_{t}$, a problem that will be examined later.

One should thus use $x_{t+1}=\left(r-b l_{t}+\varepsilon_{t}\right)+x_{t}$ or, alternatively,

$$
x_{t+1}=\left(r-b\left(x_{t}+\varsigma_{t}\right)+\varepsilon_{t}\right)+x_{t}
$$

While model [5] (or equivalently [3]) is analogous to a linear regression of $x_{t+1}$ on $x_{t}$ (to an extent that will be discussed later), model [6] is affected by an "error-in-variable" (see e.g. Fuller 1987). In practice, the latent variable $l_{t}$ is unknown and so is $\operatorname{var}\left(\varsigma_{t}\right):$ model [6] cannot be readily used instead of [3]. What are the consequences of the error-in-variable problem on detection and estimation of DD when using [3]? Under $\mathrm{H}_{0} b=0$, model [6] 
reduces to $x_{t+1}=\left(r+\varepsilon_{t}\right)+x_{t}$, i.e. model [4]. The distribution of any estimator of $b$ under $\mathrm{H}_{0}$ remains thus unaffected by the error-in-variable problem. So, although density-dependence is strictly a model concept, testing for it is equivalent to a test of dependence on resource depletion, at least in the simplistic setting considered here. As far as we know, the consequences on the estimated slope under $\mathrm{H}_{1} b>0$, i.e. the estimated strength of DD when present, remain to be explored.

\section{Methods for detecting DD in the absence of uncertainty on population size}

Model [3] $x_{t+1}=r+(1-b) x_{t}+\varepsilon_{t}$ can easily be treated by maximum likelihood, preferably conditional on $x_{1}$ which plays no role in the estimation of $b$ (Hamilton 1994 p.123; Dennis and Taper 1994 p. 209; Lebreton 2009). Because of the linear structure of the model, the Maximum Likelihood Estimator (MLE) of $1-b$ is obtained by the formula for estimating the slope of the ordinary linear regression of $x_{t+1}$ wrt $x_{t}$. This apparent simplicity is quite unfortunate, as the estimator does not benefit from the properties of the regression estimator, because the underlying statistical model is not at all the usual linear regression model: the same variable appears both as independent and dependent variable, with a shift in time, i.e. we are in a so-called autoregressive model. In figure 1 , we illustrate what happens under $\mathrm{H}_{0} b=0$ : the joint distribution of the pairs $\left(x_{t+1}, x_{t}\right)$ is aligned along the line $y=r+x$. Samples of this distribution thus have on the average a major axis with slope 1 . The regression estimate is then necessarily on the average below 1 . As a consequence, the estimator of $b$ is positively biased, i.e. the naïve approach tends to detect too often DD. Despite repeated warnings as early as the 70s (e.g. Maelzer, 1970), few people realize this bias is present indeed in the absence of uncertainty on population size (e.g. Freckleton et al. 2006, item 2 of summary) as can be seen for abscissa 0 in figure 2. Asymptotically, the estimate is not biased, just because the regression line in Figure 1 comes closer and closer to the line $y=r+x$ as the scatter of 
points become more and more elongated since $\operatorname{var}\left(x_{t}\right) \rightarrow \infty$ when $t \rightarrow \infty$ (Figure 1) while $\operatorname{var}\left(x_{t+1} / x_{t}\right)=\operatorname{var}\left(\varepsilon_{t}\right)$ remains constant. As, in studies of wild animal populations, the time series are usually fairly short, the asymptotic absence of bias offers little consolation.

Two approaches have been proposed to account for this bias:

a) Using simulation (i.e. parametric bootstrap) (Dennis and Taper 1994). Basically, one generates pseudo-samples using estimates under $\mathrm{H}_{0} b=0$ and use the appropriate percentiles of the empirical distribution of the resulting estimates of $b$ to accept or reject $\mathrm{H}_{0}$. In our model, this approach would amount to a bias correction, but Dennis and Taper (1994) use it with a slightly different, nonlinear, model in which the approach also overcomes the absence of distributional results.

b) Obtaining an expression for the bias. Lebreton (2009), reformulating results by SaintAmant (1970), develops a bias corrected t-test of $\mathrm{H}_{0} b=0$, and checks the resulting test-level is sufficiently close to the nominal $\alpha$. A bias-corrected estimate can indeed be also directly derived from the general study of the bias of the autocorrelation coefficient by Kendall (1954, in Sawa 2002) for a time series of length $T$, as $\tilde{b}=\hat{b}-\frac{4-3 \hat{b}}{T}$.

Naive approaches are still commonly used (e.g. Saether et al. 2005, Sibly et al. 2005, among others). For a particular study they can lead to grossly misleading results. In meta-analyses, the bias on the DD coefficient induces an overestimation of the prevalence of densitydependence. Based on the impossibility of long term exponential growth, one gets then a faked statistical confirmation of a plausible result! However the corrections to bias mentioned above do not appear to be a general solution to be recommended. Indeed, they largely amount to a useless statistical exercise, as the assumption of no uncertainty in $x_{t}$ is very stringent and far from met for most animal population surveys. We thus strongly recommend approaches 
not accounting for uncertainty in population size are abandoned. Handling this uncertainty is the subject of the next section.

\section{Methods accounting for uncertainty in population size}

Bulmer (1975) was the first author to formulate the linearized Gompertz model with uncertainty in population size. In this formulation, the state of the population is described by model [3] above, and only a time series of estimated log-population size $y_{t}$ is observed:

$$
x_{t+1}=r+(1-b) x_{t}+\varepsilon_{t}
$$

$$
y_{t+1}=x_{t+1}+\eta_{t+1}
$$

Bulmer (1975), in a direct treatment of the model, rewrote it as

$$
y_{t+1}=r+(1-b) y_{t}+\eta_{t+1}-(1-b) \eta_{t}+\varepsilon_{t} \quad[10]
$$

which clearly shows a further dependency over time induced by the presence of $\eta_{t}$ both in the equation for $y_{t+1}$ and that for $y_{t}$.

Neglecting it, i.e. treating $y_{t}$ by the previous regression-like method induces a severe bias (Bulmer 1975) that has rarely if ever been illustrated. Figure 2, based on simulations, clearly shows how the faked evidence for DD (i.e. the bias in $\hat{b}$ ) increases with the uncertainty in population size, over a range of realistic values of the coefficient of variation for the estimated population size.

The ad hoc tests proposed by Bulmer were criticized by Den Boer and Reddingius (1989), in particular because they are not optimal, statistically speaking. However one clearly distinguishes in the model above a state equation [7] and an observation equation [8], i.e. a linear Gaussian state-space model, which can be treated by specific methods such as the 
Kalman filter, as noted by Lebreton (1989). The Kalman filter makes it possible to obtain the

207

likelihood of the model, based on a series of observations $\left(y_{1}, y_{2}, \ldots, y_{T}\right)$, as presented in a clear and detailed way by Dennis et al. (2006). The MLE adequately exploits the statistical information available and is definitely preferable to any ad hoc estimate such as the regression estimate from the previous model or the explicit formulas proposed by Bulmer (1975). The MLE has to be obtained numerically, with, according to Dennis et al. (2006), special care because of potential multiple minima in the likelihood (see also Polansky et al. 2009). This approach removes the substantial bias that would have been present by ignoring the uncertainty in population size (Figure 2). However, the MLE is again only asymptotically unbiased, i.e. suffers from some bias for realistic sample sizes. As in the absence of sampling error, some bias corrections could be obtained from the results on the autocorrelation coefficient bias, extended to autocorrelated random errors by Sawa (2002), based on the model presentation in equation (10).

Moreover, the distribution of the ML estimate under $\mathrm{H}_{0} b=0$ cannot be easily obtained (Dennis et al. 2006). It cannot thus be used without care for a test of DD. Dennis et al. (2006) also discuss alternative estimation methods, based in particular on the first-order differences of the estimated log-population sizes $y_{t}$. Knape and de Valpine (2011) go one step further with this approach by implementing a test of DD based on the likelihood ratio statistic between model [7] and its density-independent version, obtaining its distribution under $\mathrm{H}_{0}$ $b=0$ by parametric bootstrap, exactly as Dennis and Taper (1994) did for the model without uncertainty on population size. To avoid distributional problems with the test statistics, one can use a Bayesian approach, easily implemented in WinBUGS (Lunn et al. 2000) or in R via JAGS (Plumer 2003), and, e.g., use a $95 \%$ credible interval as an admissible set of parameter values. Bayesian approaches also allow for flexibility in the functional form of the state equation - i.e. the "shape" of DD - and in the distribution of the random terms. These 
approaches currently seem the most reliable and the handiest ones, even if the underlying properties of the models, e.g., the degree to which the various parameters are separately identifiable do not depend on the estimation method and still remain often problematic.

Let us briefly examine the performance of these approaches, first by simulation. Deviance contours from simulated data (figure 3) show no evidence of local minima at the scale considered. However the estimates of $r$ and $b$ are strongly correlated for realistic lengths of the time series. They behave as the estimated intercept and slope of a regression when the dependent variable values are on a same side of the origin, to an even greater extent because of the two levels of uncertainty (process + sampling) and the dependency over time (Eq. [10]) they induce. The estimate of the process and sampling standards errors, $\operatorname{se}\left(\varepsilon_{t}\right)$ and $\operatorname{se}\left(\eta_{t}\right)$ are also highly correlated, although to a lesser degree than the estimates of $r$ and $b$, while the correlation among the two parameter subsets remain moderate. How does this translate in a real world example?

Let us use as an illustrative example the spring population surveys of Greater Snow Geese Chen caerulescens atlantica in Quebec from 1973 to 2002 (Gauthier et al . 2007). The deviance did not show local minima (Figure 4): over 1000 iterative searches starting from random initial values, only 21 did not fully converge in 1000 iterations; 15 had indeed practically converged, and 6 were still not at the global optimum. They concerned searches starting from large sampling standard error and small process standard error, a combination mimicking a short, instable, time series.

The MLEs of $r$ and $b$ are highly correlated (0.9990), and the MLE of $r, 0.3191$, seems unrealistic (Table 2, second row) as it corresponds to a $37.6 \%$ increase per year. These two features combined make the estimate of the coefficient of DD $b$ suspicious. 
The correlations between estimates (Table 1) emphasize the difficulty to separately estimate the sampling and process variance, and even more, $r$ and $b$, that are indeed nearly not separately estimable. The latter point makes practical sense: similar population trajectories can arise as the result of a high intrinsic growth rate and a strong DD, or a weaker intrinsic growth rate and a low DD (see figure 5, below). The set of four parameters nearly appears as a set of two macro-parameters, one for the deterministic trajectory, the other one for the uncertainty. Based on our simulations (Figure 3) and a variety of unpublished examples, we think this situation is general. It immediately leads to consider the use of external information to improve the identifiability. Bayesian approaches using appropriate prior distributions on the parameters appear then as a necessity, rather than just an algorithmic convenience.

An estimate of the sampling standard error will often be available, e.g., when the population size estimates come from capture-recapture (e.g. Dennis and Oten 2000, who do not account for this uncertainty in their analysis), from stratified sampling (as it is the case with the Greater snow Goose), or more directly, through replicated population sampling (Dennis et al. 2010). However, because of the high correlation with the estimated coefficient of DD, and because of the unrealistic value often obtained, as in the snow Goose example, the main target for external information is certainly the intrinsic growth rate $r$. We strongly suspect that a fair part of published examples correspond to unrealistic estimates of $r$, without being able to check, as the estimates of $r$ are rarely given in published examples because of the focus on the coefficient of DD $b$.

Assuming in the snow Goose example a Gaussian prior distribution for $r$ with mean 0.20 and standard error 0.06 (i.e. with $95 \%$ of the density between 0.12 and 0.28 ) to constrain $r$ to a realistic value for such a species (Niel and Lebreton 2005 Table 2) did improve the results (Table 1, second and third row) and provided evidence of very weak DD, with nearly no biological significance. 
The population size survey appeared as fairly precise, as the MLE of the standard error of the $\log$ population size, i.e. of its coefficient of variation is 0.0607 , comparable in magnitude to the field estimate (10\% or less, Gauthier et al. 2007 p. 1422). This fairly good precision limited the bias of the naïve regression approach (Table 1 first row), with a $95 \%$ confidence interval for the coefficient of DD encompassing 0, despite its bias. Accounting for uncertainty in population size not only removes a bias, but also increases the precision on the coefficient of DD by reducing the estimate for the process standard error.

Contrary to Dennis et al. (2006) and Knape and de Valpine (2011), we found no pervasive presence of local minima in the likelihood, although we agree that care should be exercised on this point (Knape 2008). The sampling correlation between the estimates of $r$ and $b$ and the resulting near non-identifiability is for us the main problem. Results from simulated data (Figure 3, and unpublished results on shorter time series) and a few other unpublished examples indicate similar general properties of what remains in the words of Dennis et al. (2006) a "minimal model". The improvement brought by using a prior for the intrinsic population growth rate encourages refining the model and combining it with further pieces of external information, to improve its moderate performance. It gives a central role to Bayesian approaches, which also bring a great deal of flexibility. We do not think reasonable to consider as a fair evidence for DD a value of $\hat{b}$ significantly differing from 0 without checking if the associated $\hat{r}$ value makes sense. We currently recommend as a minimal step a careful look at the estimated intrinsic growth rate, and the use of a prior for $r$ based on comparative demography approaches (e.g; Niel and Lebreton 2005).

\section{From a minimal to realistic models}

Besides the difficulties just mentioned, the minimal model above may be inappropriate in practice for a variety of reasons (Lebreton 2009). The most prominent issues are: 
- Environmental covariates have to be taken into account to reduce the residual standard error and enhance power, and, if negatively autocorrelated, to distinguish their effect from that of DD (e.g. Lebreton 1990).

- Age structure, e.g. with delayed recruitment, may require considering dependency over several time steps in components of population size.

- Further structure such as spatial cells may have to be considered, e.g. to model local

Neglecting any of these issues may result in a model misspecification, and thus in biases of the estimate of the intensity of DD and the corresponding tests or diagnostics of DD.

While in the original presentation by Bulmer (1975) the minimal model was presented as quite a specific statistical object, the state-space model point of view opens a number of In all such models, the main potential bias of the slope for DD is removed by incorporating the population size uncertainty in the observation equation. However, the slope remains 
biased for finite sample sizes to an unknown degree. The likelihood for such models can be multimodal (Polansky et al. 2009) and the MLE as a consequence often difficult to obtain.

Parametric bootstrap for such realistic models (e.g., with age structure, with covariates) may be tedious and remain thus confidential until a specialized user-friendly piece of software is produced. REML approaches based on first order differences found promising by Dennis et al (2006) could be useful, but have still to be investigated in such a more complex setting. A Bayesian approach seems the most straightforward treatment. As an alternative to MCMC algorithms, the posterior distributions can be obtained through numerical integration (de Valpine and Hastings, 2002), and explicitly in simple cases under Gaussian distributions.

\section{An overview of methods based on time series of estimated population sizes}

The first clear recommendation is to abandon all methods not accounting for uncertainty in population size, as they are unavoidably biased when used with real world, "noisy", estimates of population size. This conclusion, although developed here based on a simple model, applies to any such approach such as the regression of "observed growth rate" $y_{t+1}-y_{t}$ vs observed population size $y_{t}$ as well illustrated by Freckleton et al. (2006 Figure 1c). The resulting bias is particularly vicious in meta-analyses, in which the estimated proportion of populations subject to DD will unavoidably be overestimated. This was suspected in the analysis by Brook and Bradshaw (2006) by Lebreton (2009) and demonstrated for these same data by Knape and De Valpine (2011). Brook and Bradshaw conclude to DD in more than $75 \%$ of the cases studied, while, accounting for uncertainty in population size estimates and using a Bayesian approach, Knape and de Valpine conclude to significant DD in only $16 \%$ of the case studied. Similarly, based on reliable tools, Jamieson and Brooks (2004) conclude to weak to moderate DD among American ducks. We still suspect part of these conclusions would not resist a close examination of the resulting intrinsic growth rate estimates. 
The state-space model formulation provides a sensible approach to account for uncertainty in population size (Dennis et al. 2006) and to develop specific models accounting for environmental covariates, age structure etc.., and testing thus for DD in presence of such potentially confounding effects.

Two intermingled issues severely limit the potential of such models if used by themselves:

1) Numerical issues (multiple maxima to the likelihood or sensitivity to priors, depending on the type of algorithms used) and problems of bias associated with finite sample size which currently make such models difficult to use without specific help from a specialist;

2) Low power and identifiability problems as a result of the shortness of the time series and the two-level uncertainty, in particular if further complexities are brought in the model, at an unavoidable cost in terms of number of parameters to be estimated. We would rarely expect evidence for DD with fewer than 30 points and we think external information on the intrinsic growth rate has to be seriously considered in any such analysis unless the grail to remain inaccessible.

The situation is somewhat similar to that of capture-recapture models for closed populations, whose status rapidly moved in the last few years from an innocuous fairly standard approach to that of delicate, specialized models with severe issues of bias and robustness (Link 2003).

We will come back in greater detail in the general discussion to the potential of state-space models.

\section{DETECTING AND ESTIMATING DENSITY DEPENDENCE BASED ON TRAITS}

By contrast, one clear type of evidence for DD concerns the response of life history traits to changes in population size. Surprisingly, most such studies are observational correlative 
studies relating particular traits to observed changes in population size over time. Some qualify as quasi-experiments, as based on a clear population crash or explosion (e.g. Aslhey et al. 1998). Despite the current development of experimental ecology, few really manipulative experiments of densities have ever taken place. The few such studies with nest boxes (Torok and Toth, 1988, Alatalo and Lundberg 1984), although quite convincing, do not take full advantage of an experimental setting, notably in terms of block design and replication (see also Newton 1994). Bartmann et al. (1992) provide a good example of an experimental study of density dependent compensatory mortality in the Mule Deer with several replicates.

The traits investigated in a search for DD can be morphological, such as body weight (e.g. Gaillard et al. 1996), and are then relatively simple to study. Demographic traits such as fecundity can be studied in a similar fashion (e.g. Arcese and Smith 1988). Demographic traits less directly measurable, such as survival, require more sophisticated approaches such as capture-recapture models (Lebreton et al. 1992, Roe Deer C.capreolus; Catchpole et al. 2000, Soay Sheep). A full review of studies relating estimated population size to life history traits, whether demographic or not, is beyond the purpose of this paper. Bonenfant et al. (2009) provide a broad review of density-dependence in mammals, discussing trait response to density, as well as different responses by different segments of the population, such as males and females or age classes. Newton (1998) reviews a number of trait - density or trait resource relationships in birds.

In all analyses of traits, one has to deal with some kind of linear model between the density or population size (possibly transformed, to log, to discrete categories...), $x_{t}$, and the trait at time $t$ in individual $j, z_{t j}$ :

$$
z_{t j}=a+b x_{t}+\varepsilon_{t}+\eta_{t j}
$$


The model may have to incorporate further effects, as the two-level sampling (individuals and years) raises some specific issues (whether for instance the same individuals are sampled over several years or not). Environmental covariates can easily be incorporated as additive effects (Lebreton et al. 1992, Roe Deer example; Catchpole et al. 2000 for survival; Crespin et al. 2006 for recruitment; Gaillard et al. 1996 for morphometric traits). The prominent point common to all approaches is again that the density is always a proxy for something else and is always known with some uncertainty, i.e. we are again faced with an error-in-variable problem. Model [11] should be accompanied by an observation equation $y_{t}=x_{t}+\varsigma_{t}$. When using the regression model $z_{t j}=a+b y_{t}+\varepsilon_{t}+\eta_{t j}$, attenuation of the slope estimate towards 0 (McArdle 2003) is unavoidable. Clearly, any test on the slope will be conservative, i.e., contrary to methods based on population size, the presence and intensity of DD is not overestimated, which is good news. As we usually have not much control on the uncertainty in population size, several possibilities are available to account for the error-in-variable problem, of which most remain to be explored:

- Use an error-in-variable model (Fuller 1987); however, all such models are weakly identifiable, through stringent distributional assumptions, as they exploit differences in distribution in the $\varepsilon$ and the $\eta$ terms to estimate their relative effect on the variation in the response variable;

- Correct for bias. Barker et al. (2002), develop such an approach in the context of capture-recapture survival models. They develop two examples with seabird data that provide no evidence for DD.

- Use information on the precision of population estimates, i.e. in the notation above an estimate (or a prior distribution) for $\operatorname{var}\left(\varsigma_{t}\right)$ to improve identifiability in the error-invariable model. 
- Recast the capture-recapture model as a state-space model (Gimenez et al. 2007) and consider besides the observation equations needed for representing the recapture process an observation equation $y_{t}=x_{t}+\varsigma_{t}$. In such an approach, survival for instance is represented as state equations made of Bernoulli (0/1) random variables, and the detection/recapture process as observation equations also made of 0/1 random variables.

- Use instrumental variables, a tool commonly used in econometrics (e.g. Stock 2001). An instrumental variable will be in our context a variable uncorrelated with the random term for estimated population size, $\varsigma_{t}$ and correlated with true population size. Alternative, independent estimates of population size could be good candidates (for an example using multiple surveys, see Fromentin et al. 2001).

The impact of the error-in-variable problem is clearly relatively limited, and, although some statistical care should be exercised, notably for what concerns the use of capture-recapture methodology, the assessment of DD based on traits is relatively straightforward.

\section{DISCUSSION}

The first part of our review, on methods for detecting DD based on population size surveys, leads first to several straightforward recommendations, to avoid the pervasive risk of overestimating the strength of DD. The first one is that methods neglecting uncertainty in population size should definitely be abandoned. The second is that the Bayesian approach to simple state-space models, such as the linearized Gompertz model, accounting for uncertainty in population size should be used, with some caution because of practical difficulties. We recommend also a reasonable prior based on external comparative information is used for the population growth rate. Even when these difficulties are correctly handled, which may require the assistance of an applied statistician with good knowledge of these models and their tricks, 
the simplest models may remain strongly misspecified because they neglect a number of features such as environmental covariates, age structure, particular functional form of DD, etc... Moving away from such minimal models is alike moving from simple regression to more sophisticated linear or nonlinear models: one cannot avoid a trade-off between the improvement brought by a better adaptation of a more complex model to the data (such as a decrease in residual variance), and the loss of precision implied by a greater number of parameters: modeling remains the art of oversimplification. Again, in this context, a Bayesian approach is probably the preferable approach, with caution exercised. In spite of their statistical and practical difficulties, methods based on population size surveys remain very attractive as they immediately translate into population projections. They are often the only practicable analyses of DD when population size surveys only are available, a common situation for managed wildlife populations. There is however little power for time series with fewer than, say, 30 time steps, i.e., in most cases, years. One has to recall that when the power drops down to values close to the test level $\alpha$, the analysis boils down to deciding for DD or no DD on the basis of a random number.

When moving from methods based on population size surveys to methods for detecting DD on traits, one is, in some sense, moving from pattern to process (Swihart et al. 2002). The most straigthforward methods for traits, such as using density as a covariate in capturerecapture models, are conservative, i.e. tend to underestimate the strength of DD, which means that the evidence for DD is reliable when present. Several perspectives to correct for the conservative effect of uncertainty in population size will probably be explored in the near future. The most promising is the use of the state-space formulation of capture-recapture models (Gimenez et al. 2007), completed by an observation equation for population size to properly model DD. While they can thus be used more confidently than approaches based on population size surveys, methods for DD in traits do not easily lead to population projections. 
469 Even when the traits under study are demographic, they can only be translated into projections through a projection model such as a matrix model. Nevoux et al. (2011) examine the consequences of DD in different traits of the Mauritius Kestrel Falco punctatus by integrating these different traits and their DD relationships into a nonlinear discrete time model, studied independently from the trait analyses. In the absence of correction for attenuation, the results of such models should be looked at with a critical eye. Moreover, deterministic models do not account for the interplay of DD and various forms of stochasticity.

As the state-space formulation can easily encompass projection models (e.g. Gauthier et al. 2007) and as it brings decisive advantages for assessing DD whether from population surveys or in studies of traits, it is clear that the future of DD modeling lies with state-space modeling.

One can easily foresee what could be a state-space model incorporating DD in a wildlife population, based on integrated monitoring covering both population size surveys and individual longitudinal data (i.e. capture-mark-recapture data in the broad sense). Such a model has to combine different types of state equations, with possibly at some stage the need to account for the lack of independence between the marked individuals and the overall population:

- Multinomial distributions of individual trajectories on a Markov chain (reducing to Bernoulli equations in the case of survival), with parameters possibly dependent on population size, i.e. density-dependent;

- Equations to iterate a population vector submitted to demographic stochasticity.

The corresponding observation equations are:

- Discrete variables such as Bernoulli for the capture-recapture process; 
- Equations for the uncertainty in the observation for population size (as a vector or total number).

493

494

495

496

497

498

499

500

501

502

503

504

505

506

507

508

509

510

A model on these lines is proposed by Abadi et al. (submitted). Rotella et al ; (2009) go also one step in this direction by examining Weddell Seal population size estimates derived together with demographic parameters from a capture-recapture analysis within a stochastic Gompertz model. The demographic parameters are however not examined for DD and the estimated intrinsic growth rate is not given.

The first consequence of this evolution is that detecting and estimating DD has already started moving from a push-button procedure to a full size modeling exercise. A second consequence is that the gap between projection models and statistical models is progressively filled. In such a promising state of the art one may also expect a progressive integration of models based on mechanism at the individual levels, in the spirit of individual-based models, with state-space models derive from the classical phenomenological approach to population dynamics reviewed here (e.g., Stephens et al. 2002).

This evolution is fortunate as DD as a complex biological phenomenon has no reason to be uniformly reduced to a simple omnibus model, apart from a theoretical point of view that makes the logistic growth curve (Verhuslt 1838) so useful. We have to think of DD as a complex biological process interacting with other processes rather than in terms of a simple equation: modern statistical and modeling tools make such a synthesis within reach. 
We thank Gilles Gauthier for having authorized me to use the Greater Snow Goose Spring survey data. We thank Bret Collier and Bob Steidl for helpful comments on an earlier version.

\section{LITERATURE CITED}

515

516

Abadi, F., O. Gimenez, W. Jakober, R. Arlettaz, and M. Schaub. Submitted. Estimating the strength of density-dependence in the presence of observation errors using integrated population models.

Alatalo, R. V., and A. Lundberg. 1984. Density-Dependence in breeding success of the pied flycatcher (Ficedula hypoleuca). Journal of Animal Ecology 53:969-977.

Andrewartha, H. G., and L. C. Birch. 1954. The distribution and abundance of animals. University of Chicago Press, Chicago.

Arcese, P., and J. N. M. Smith. 1988. Effects of population density and supplemental food on reproduction in song sparrows. Journal of Animal Ecology 57:119-136.

Ashley, E. P., G. B. McCullough, and J. T. Robinson. 1998. Morphological responses of white-tailed deer to a severe population reduction Canadian Journal of Zoology 76:15.

Barker, R., D. Fletcher, and P. Scofield. 2002. Measuring density dependence in survival from mark-recapture data. Journal of Applied Statistics 29:305-313.

Bartmann, R. M., G. C. White, and L. H. Carpenter. 1992. Compensatory mortality in a Colorado Mule Deer population. Wildlife Monographs 121: 1-39.

Berryman, A. A. 2004. Limiting factors and population regulation. Oikos 105:667-670.

Bonenfant, C., J. M. Gaillard, T. Coulson, M. Festa-Bianchet, A. Loison, M. Garel, L. E. Loe, P. Blanchard, N. Pettorelli, N. Owen-Smith, J. Du Toit, and P. Duncan. 2009. Empirical Evidence of Density-Dependence in Populations of Large Herbivores. Pages 313-357 in H. Caswell, editor. Advances in Ecological Research, Vol 41. 
Brook, B. W., and C. J. A. Bradshaw. 2006. Strength of evidence for density dependence in abundance time series of 1198 species. Ecology 87:1445-1451.

Bulmer, M. G. 1975. The statistical analysis of density-dependence. Biometrics 31:901-911.

Caswell, H. 2001. Matrix population models. 2nd edition, in press edition. Sinauer, Sunderland, Mass.

Catchpole, E. A., B. J. T. Morgan, T. N. Coulson, S. N. Freeman, and S. D. Albon. 2000. Factors influencing Soay sheep survival. Journal of the Royal Statistical Society Series C-Applied Statistics 49:453-472.

Crespin, L., M. P. Harris, J.-D. Lebreton, M. Frederiksen, and S. Wanless. 2006. Recruitment to a seabird population depends on environmental factors and on population size. Journal of Animal Ecology 75:228-238.

De Valpine, P. 2002. Review of methods for fitting time-series models with process and observation error and likelihood calculations for nonlinear, non-Gaussian state-space models. Bulletin Of Marine Science 70:455-471.

De Valpine, P., and A. Hastings. 2002. Fitting population models incorporating process noise and observation error. Ecological Monographs 72:57-76.

Dennis, B. and M. R. M. Otten. 2000. Joint effects of density dependence and rainfall on abundance of San Joaquin kit fox. Journal of Wildlife Management 64: 388-400.

Dennis, B., and M. L. Taper. 1994. Density-dependence in time-series observations of natural populations - Estimation and testing. Ecological Monographs 64:205-224.

Dennis, B., J. M. Ponciano, S. R. Lele, M. L. Taper, and D. F. Staples. 2006. Estimating density dependence, process noise, and observation error. Ecological Monographs 76:323-341.

Diekmann, O. 2005. On the Mathematical synthesis of physiological and behavioural mechanisms and population dynamics. Centre for Mathematics and Computer Science. 
Freckleton, R. P., A. R. Watkinson, R. E. Green, and W. J. Sutherland. 2006. Census error and the detection of density dependence. Journal of Animal Ecology 75:837-851.

Fromentin, J. M., R. A. Myers, O. N. Bjornstad, N. C. Stenseth, J. Gjosaeter, and H. Christie. 2001. Effects of density-dependent and stochastic processes on the regulation of cod populations. Ecology 82:567-579.

Fuller, W. A. 1987. Measurement error models. Wiley, New-York.

Gaillard, J. M., D. Delorme, J. M. Boutin, G. VanLaere, and B. Boisaubert. 1996. Body mass of roe deer fawns during winter in 2 contrasting populations. Journal of Wildlife Management 60:29-36.

Gauthier, G., P. Besbeas, B. J. T. Morgan, and J.-D. Lebreton. 2007. Population growth in Snow Geese: A modeling approach integrating demographic and survey information. Ecology 88:1420-1429.

Gimenez, O., V. Rossi, R. Choquet, C. Dehais, B. Doris, H. Varella, J.-P. Vila, and R. Pradel. 2007. State-space modelling of data on marked individuals. Ecological Modelling 206:431-438.

Guthery, F. S., and J. H. Shaw. 2012. Density-Dependence: applications in widlife management. Journal of Wildlife Management In press.

Hamilton, J. D. 1994. Time series analysis. Princeton, NJ, Princeton University Press.

Jacobson, A. R., A. Provenzale, A. von Hardenberg, B. Bassano and M. Festa-Bianchet. 2004. Climate forcing and density-dependence in a mountain ungulate population. Ecology 85: $1598-1610$.

Jamieson, L.E. and S. P. Brooks. 2004. Density dependence in North American ducks. Animal Biodiversity and Conservation 27:113-128.

Knape, J. 2008. Estimability of density-dependence in models of time-series data. Ecology 89:2994-3000.

Knape, J., and P. De Valpine. In revision. Are patterns of density dependence in the Global Population Dynamics Database driven by observation errors? 
Krebs, C. J. 1995. Two paradigms of population regulation. Wildlife Research 22:1-10.

Lack, D. 1954. The natural regulation of animal numbers. Oxford University Press, Oxford.

Lebreton, J.-D. 1989. Statistical methodology for the study of animal populations. Bull. International Statistical Institute 53:267-282.

Lebreton, J.-D. 1990. Modelling density-dependence, environmental variability, and demographic stochasticity from population counts: an example about Wytham Great tits. Pages 89-102 in J. Blondel, A. Gosler, J.-D. Lebreton, andR. McCleery, editors. Population biology of passerine birds an integrated approach. Springer,Berlin.

Lebreton, J.-D. 2009. Assessing density-dependence: where are we left? Modeling demographic processes in marked populations. T. D. L., E. G. Cooch and M. J. Conroy, Springer. 3: 19-42.

Lebreton, J.-D., K. P. Burnham, J. Clobert, and D. R. Anderson. 1992. Modeling survival and testing biological hypotheses using marked animals: a unified approach with case studies. Ecological Monographs 62:67-118.

Link, W. A. 2003. Nonidentifiability of population size from capture-recapture data with heterogeneous detection probabilities. Biometrics 59:1123-1130.

Lunn, D.J., A. Thomas, A., N. Best, and D. Spiegelhalter (2000) WinBUGS -- a Bayesian modelling framework: concepts, structure, and extensibility. Statistics and Computing, $10: 325-337$.

Maelzer, D. A. 1970. The regression of $\log \left(\mathrm{N}_{\mathrm{t}+1}\right)$ on $\log \left(\mathrm{N}_{\mathrm{t}}\right)$ as a test of density-dependence: an exercise with computer constructed density independent populations. Ecology $51: 810-822$.

Malthus, T. R. 1798. An essay on the principle of population. Pages 4-16 in G. Hardin, editor. Population, evolution, and birth control: a collage of controversial ideas (1964). Freeman,San Francisco.

Maynard Smith, J. 1965. Professor J. B. S. Haldane, FRS. Nature 206:239-240. 
McArdle, B. H. 2003. Lines, models, and errors: Regression in the field. Limnology and Oceanography 48:1363-1366.

Murdoch, W. W. 1994. Population regulation in theory and practice. Ecology 75:271-287.

Nevoux, M., O. Gimenez, D. Arlt, M. Nicoll, C. Jones, and K. Norris. 2011. Population regulation of territorial species: both site dependence and interference mechanisms matter. Proceedings of the Royal Society B-Biological Sciences 278:2173-2181.

Newton, I. 1994. Experiments on the limitation of bird breeding densities - a review. Ibis $136: 397-411$.

Newton, I. 1998. Population limitation in birds. Academic Press, London.

Pasinelli, G., M. Schaub, G. Hafliger, M. Frey, H. Jakober, M. Muller, W. Stauber, P. Tryjanowski, J. L. Zollinger and L. Jenni. 2011. Impact of density and environmental factors on population fluctuations in a migratory passerine. Journal of Animal Ecology 80: $225-234$.

Plummer, M. 2003. JAGS: A Program for Analysis of Bayesian Graphical Models Using Gibbs Sampling, . ISSN 1609-395X. Proceedings of the 3rd International Workshop on Distributed Statistical Computing DSC 2003, March 20-22, , Vienna, Austria.

Polansky, L., P. de Valpine, J. O. Lloyd-Smith, and W. M. Getz. 2009. Likelihood ridges and multimodality in population growth rate models. Ecology 90: 2313-2320.

Reddingius, J., and P. J. Denboer. 1989. On the stabilization of animal numbers - problems of testing. 1. Power estimates and estimation errors. Oecologia 78:1-8.

Rotella, J. J., W. A. Link, J. D. Nichols, G. L. Hadley, R. A. Garrott, and K. M. Proffitt. 2009. An evaluation of density-dependent and density-independent influences on population growth rates in Weddell seals. Ecology 90: 975-984.

Saether, B. E., R. Lande, S. Engen, H. Weimerskirch, M. Lillegard, R. Altwegg, P. H. Becker, T. Bregnballe, J. E. Brommer, R. H. McCleery, J. Merila, E. Nyholm, W. Rendell, R. R. Robertson, P. Tryjanowski, and M. E. Visser. 2005. Generation time and temporal scaling of bird population dynamics. Nature 436:99-102. 
Sæther, B.-E., S. Engen, R. Lande, C. Both, and M. E. Visser. 2002. Density dependence and stochastic variation in a newly established population of a small songbird. Oikos 99:331-337.

Sibly, R. M., D. Barker, M. C. Denham, J. Hone, and M. Pagel. 2005. On the regulation of populations of mammals, birds, fish, and insects. Science 309:607-610.

Stenseth, N. C., H. Viljugrein, T. Saitoh, T. F. Hansen, M. O. Kittilsen, E. Bølviken, and F. Glöckner. 2003. Seasonality, density dependence, and population cycles in Hokkaido voles. Proceedings of the National Academy of Sciences USA 100:11478-11483.

Stephens, P. A., F. Frey-Roos, W. Arnold, and W. J. Sutherland. 2002. Model complexity and population predictions. The alpine marmot as a case study. Journal of Animal Ecology 71:343-361.

Swihart, R. K., J. B. J. Dunning, and P. M. Waser. 2002. Gray matters in ecology: dynamics of pattern, process and scientific progress. Bulletin of the Ecological Society of America 83:149-155.

Torok, J., and L. Toth. 1988. Density dependence in reproduciton of the collared flycatcher (Ficedula alboicollis) at high population levels. Journal of Animal Ecology 57:251258.

Tuljapurkar, S. 1990. Population dynamics in variable environments. Springer, New-York.

Turchin, P. 2001. Does population ecology have general laws? Oikos 94:17-26.

Verhulst. 1838. Notice sur la loi que suit la population dans son accroissement (English translation). in E. J. Kormondy, editor. (1965) Readings in ecology. PrenticeHall,Englewood Cliffs, New-Jersey.

Wang, G., N. T. Hobbs, S. Twombly, R. B. Boone, A. W. Illius, I. J. Gordon, and J. E. Gross. 2009. Density dependence in northern ungulates: interactions with predation and resources. Population Ecology 51: 123-132. 
667 Table 2: sampling correlations between Maximum Likelihood Estimates of the parameters of 668 the stochastic Gompertz model for the Greater Snow Goose data

\begin{tabular}{|l|c|c|c|c|}
\hline $\begin{array}{l}\text { Parameters } \\
\text { (as rows and } \\
\text { columns) }\end{array}$ & $\begin{array}{c}\text { Sampling } \\
\text { standard error } \\
\mathrm{se}\left(\eta_{t}\right)\end{array}$ & $\begin{array}{c}\text { Process } \\
\text { standard error } \\
\mathrm{se}\left(\varepsilon_{t}\right)\end{array}$ & $\begin{array}{c}\text { Intrinsic } \\
\text { growth rate } \\
\mathrm{r}\end{array}$ & $\begin{array}{c}\text { Coefficient } \\
\text { of DD } \\
\mathrm{b}\end{array}$ \\
\hline $\mathrm{se}\left(\eta_{t}\right)$ & 1.0000 & -0.7063 & 0.2955 & 0.2910 \\
\hline $\mathrm{se}\left(\varepsilon_{t}\right)$ & -0.7063 & 1.0000 & -0.2947 & -0.2900 \\
\hline $\mathrm{r}$ & 0.2955 & -0.2947 & 1.0000 & 0.9989 \\
\hline $\mathrm{b}$ & 0.2910 & -0.2900 & 0.9989 & 1.0000 \\
\hline
\end{tabular}

669

670 
672 Table 2: Estimation of parameters $r, b, \operatorname{var}\left(\varepsilon_{t}\right)$, and $\operatorname{var}\left(\eta_{t}\right)$ of the density dependent

673 model $x_{t+1}=r+(1-b) x_{t}+\varepsilon_{t}$ with uncertainty on log-population size represented as

$674 y_{t}=x_{t}+\eta_{t}$, applied to spring population surveys of the greater Snow Goose (data from

675 Gauthier 2007).

676 First row: naïve regression approach on log population size, neglecting uncertainty in

677 population size.

678 Second row: Maximum Likelihood estimation of the model accounting for uncertainty in

679 population.

680 Third row: Bayesian approach of that same model with a Gaussian prior distribution for $r$

681 with mean 0.20 and standard error 0.06 and uninformative priors for the other parameters.

682 Fourth row: Bayesian approach of that same model with a Gaussian prior distribution for $r$ 683 with mean 0.20 and standard error 0.06 , explicit posterior normal distribution

\begin{tabular}{|c|c|c|c|c|c|c|}
\hline $\begin{array}{l}\text { Parameter } \\
\text { Method }\end{array}$ & $\begin{array}{l}\text { Process } \\
\text { Standard } \\
\text { Error } \\
\sqrt{\operatorname{var}\left(\varepsilon_{t}\right)}\end{array}$ & $\begin{array}{l}\text { Observation } \\
\text { Standard } \\
\text { Error } \\
\sqrt{\operatorname{var}\left(\eta_{t}\right)}\end{array}$ & $\begin{array}{l}\text { Growth } \\
\text { rate } \\
r\end{array}$ & $\begin{array}{l}\text { Coef- } \\
\text { ficient of } \\
\text { DD } \\
b\end{array}$ & $\begin{array}{l}\text { Corr } \\
(\hat{r}, \hat{b})\end{array}$ & $\begin{array}{c}\text { Deter- } \\
\text { ministic } \\
\text { equilibriu } \\
\mathrm{m} \\
\hat{\mathrm{r}} / \hat{b}\end{array}$ \\
\hline $\begin{array}{l}\text { Naïve regression } \\
\text { neglecting uncertainty in } \\
\text { population size }\end{array}$ & 0.1398 & - & $\begin{array}{c}0.5044 \\
{[-0.5446,} \\
1.5533]\end{array}$ & $\begin{array}{c}0.0357 \\
{[-0.0469} \\
0.1184]\end{array}$ & 0.9988 & 14.12 \\
\hline $\begin{array}{l}\text { Model with uncertainty in } \\
\text { population size: } \\
\text { Maximum Likelihood } \\
\text { Estimate and, in brackets, } \\
95 \% \text { CI }\end{array}$ & 0.1041 & 0.0607 & $\begin{array}{c}0.3191 \\
\\
{[-0.2731} \\
0.9112]\end{array}$ & $\begin{array}{c}0.0210 \\
\\
{[-0.0258,} \\
0.0678]\end{array}$ & 0.9990 & 15.21 \\
\hline $\begin{array}{l}\text { idem, Bayesian approach } \\
\text { with prior constraining } r \text { : } \\
\text { posterior distribution of } \\
\text { parameters, } \\
\text { Mean and, in brackets, } 2.5 \\
\% \text { and } 97.5 \% \text { quantiles } \\
\text { (MCMC) }\end{array}$ & 0.1210 & 0.0528 & $\begin{array}{c}0.2022 \\
{[0.0865} \\
0.2413]\end{array}$ & $\begin{array}{c}0.0118 \\
{[0.0019} \\
0.0151]\end{array}$ & 0.931 & 17.19 \\
\hline $\begin{array}{l}\text { Idem, based on normal } \\
\text { distribution }\end{array}$ & 0.1022 & 0.0628 & $\begin{array}{c}0.2024 \\
{[0.1201,} \\
0.2847]\end{array}$ & $\begin{array}{c}0.0118 \\
{[00049,} \\
0.0187]\end{array}$ & 0.951 & 17.15 \\
\hline
\end{tabular}


Figure 1: Current log population size $x_{t+1}$ vs previous one $x_{t}$ based on the density independent model $x_{t+1}=r+x_{t}+\varepsilon_{t}$, under $r=0.3$ and $\operatorname{var}\left(\varepsilon_{t}\right)=0.64$. From top to bottom, simulations over 5, 10 and 20 time occasions, respectively. The regression line of $x_{t+1}$ vs $x_{t}$ (dotted lines) progressively converges to the line $x_{t+1}=r+x_{t}$ (plain lines) as $\operatorname{var}\left(x_{t}\right) \rightarrow \infty$, i.e. the regression slope progressively converges to 1 . For all finite sample sizes, this approach points to a model $x_{t+1}=r+(1-b) x_{t}+\varepsilon_{t}$ with $b>0$, i.e. to density-dependence.

Figure 2: Estimated strength of density dependence (ordinate) in absence of density neglected. The bias in the estimated strength of DD strongly increases with the uncertainty in population size.

Simulated data based on the density independent model $x_{t+1}=r+x_{t}+\varepsilon_{t}$ with uncertainty on $\log$-population size represented as $y_{t}=x_{t}+\eta_{t}$. The estimated strength of DD (ordinate) is the Maximum Likelihood estimate of $b$ in the model $y_{t+1}=r+(1-b) y_{t}+\varepsilon_{t}$ over 30 time steps in which $y$ is treated as the "true" log population size. In abscissa, $s e\left(y_{t} / x_{t}\right)=s e\left(\eta_{t}\right)$ is the coefficient of variation of the estimated population size. Dotted line: Mean estimate $\overline{\hat{b}}(5000$ replicates each), vertical lines; $95 \%$ limits of the 5000 replicates. 
Figure 3: Deviance (-2 x log-likelihood) contours of simulated trajectories of increasing number of time steps $(\mathrm{T}=10,20,50$ and 100$)$ of the density dependent model $x_{t+1}=r+(1-b) x_{t}+\varepsilon_{t}$ with uncertainty on log-population size represented as $y_{t}=x_{t}+\eta_{t}$. The parameters used in the simulations are $r=0.1, b=0.3, s e\left(\varepsilon_{t}\right)=0.2, s e\left(\eta_{t}\right)=0.4$. The deviance is represented under $s e\left(\varepsilon_{t}\right)=0.2, s e\left(\eta_{t}\right)=0.4$ as a function of $b$ (in abscissa, from -0.3 to 0.7 ) and $r$ (in ordinate, from -0.5 to 0.7 ). The contours clearly show that in presence of uncertainty on population size a) the likelihood and the maximum likelihood estimates are well behaved in the absence of model misspecification; b) for realistic number of time steps ( $<50$ ), the estimates of $r$ and $b$ will be strongly positively correlated, making it difficult to distinguish between low intrinsic growth rate and light density-dependence vs high intrinsic growth rate and strong density-dependence.

Figure 4: Profile Deviance for parameter $r$ of the density dependent model $x_{t+1}=r+(1-b) x_{t}+\varepsilon_{t}$ with uncertainty on log-population size represented as $y_{t}=x_{t}+\eta_{t}$, applied to spring population surveys of the greater Snow Goose (data from Gauthier 2007). For each value of $r$ the deviance is maximized with respect to the three other parameters $b$, $\operatorname{var}\left(\varepsilon_{t}\right)$, and $\operatorname{var}\left(\eta_{t}\right)$.

Figure 5: reconstructed log spring population size for the Greater Snow Goose, based on the relationship $x_{t+1}=r+(1-b) x_{t}$. Plain line: MLEs of $r$ and $b$; dotted line: Estimates under a Gaussian prior distribution for $r$ with mean 0.20 and standard error 0.06 . The ability to discriminate among pairs of values $(r, b)$ is obviously very low, as different pairs of values give closely similar curves. 
Figure 1
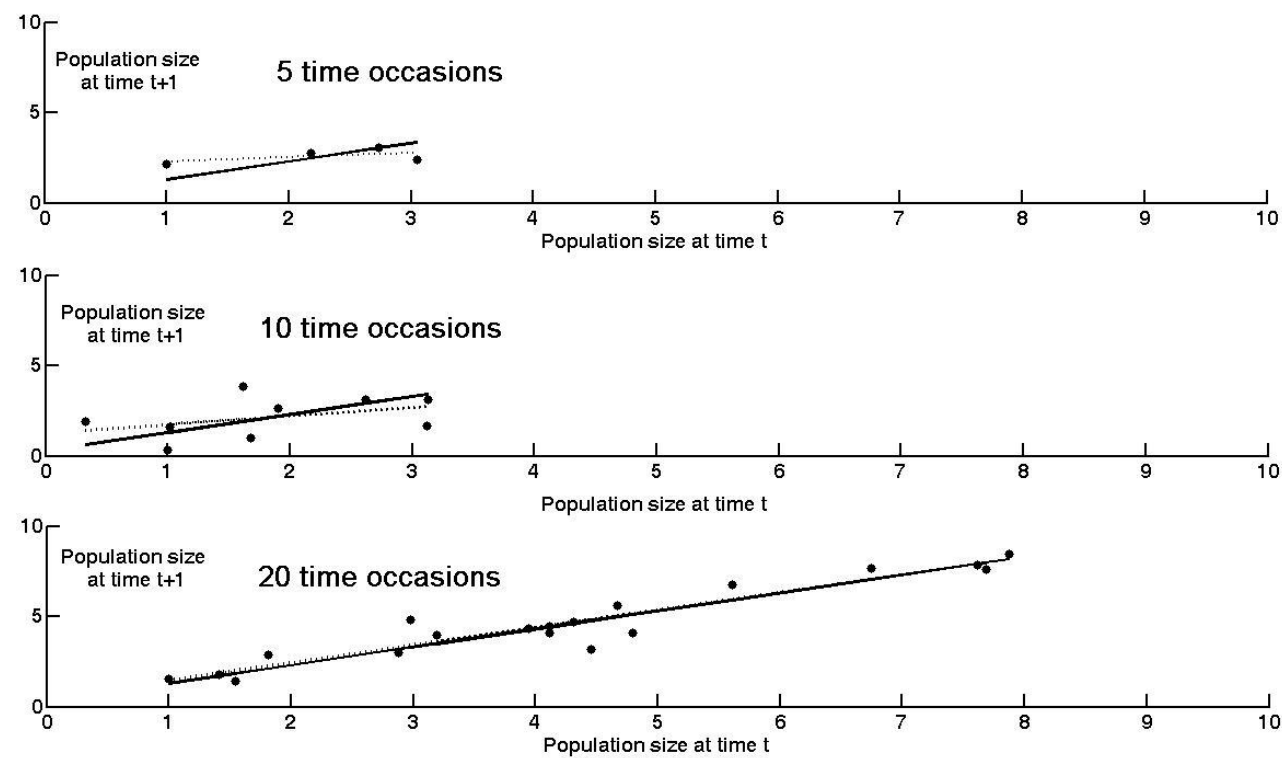
Figure 2

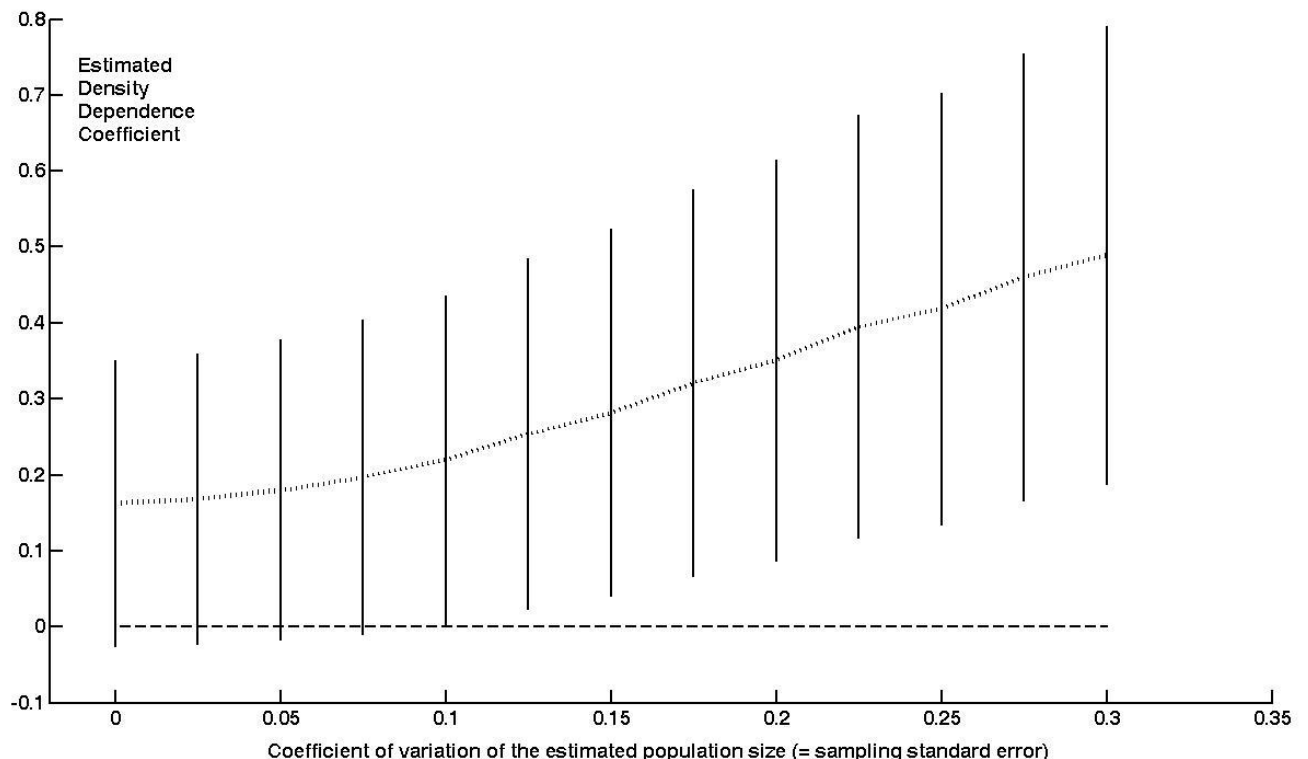


Figure 3
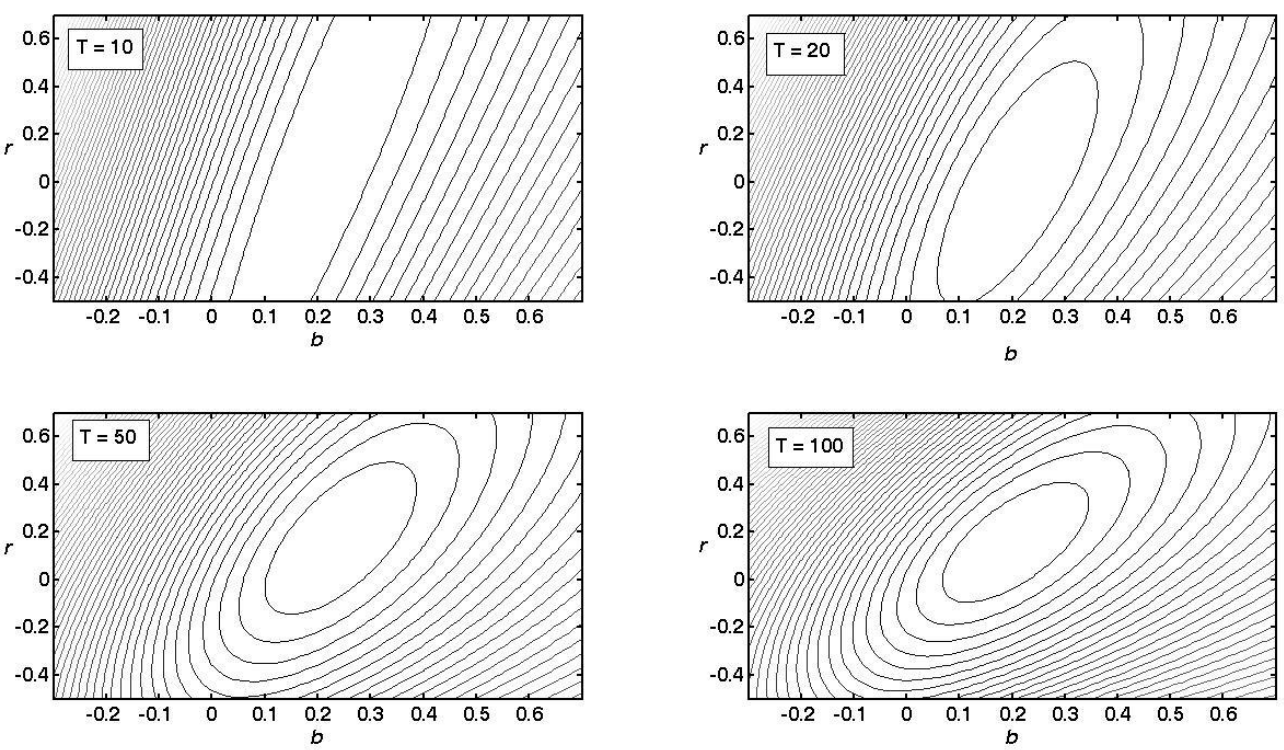

736

737 
Figure 4

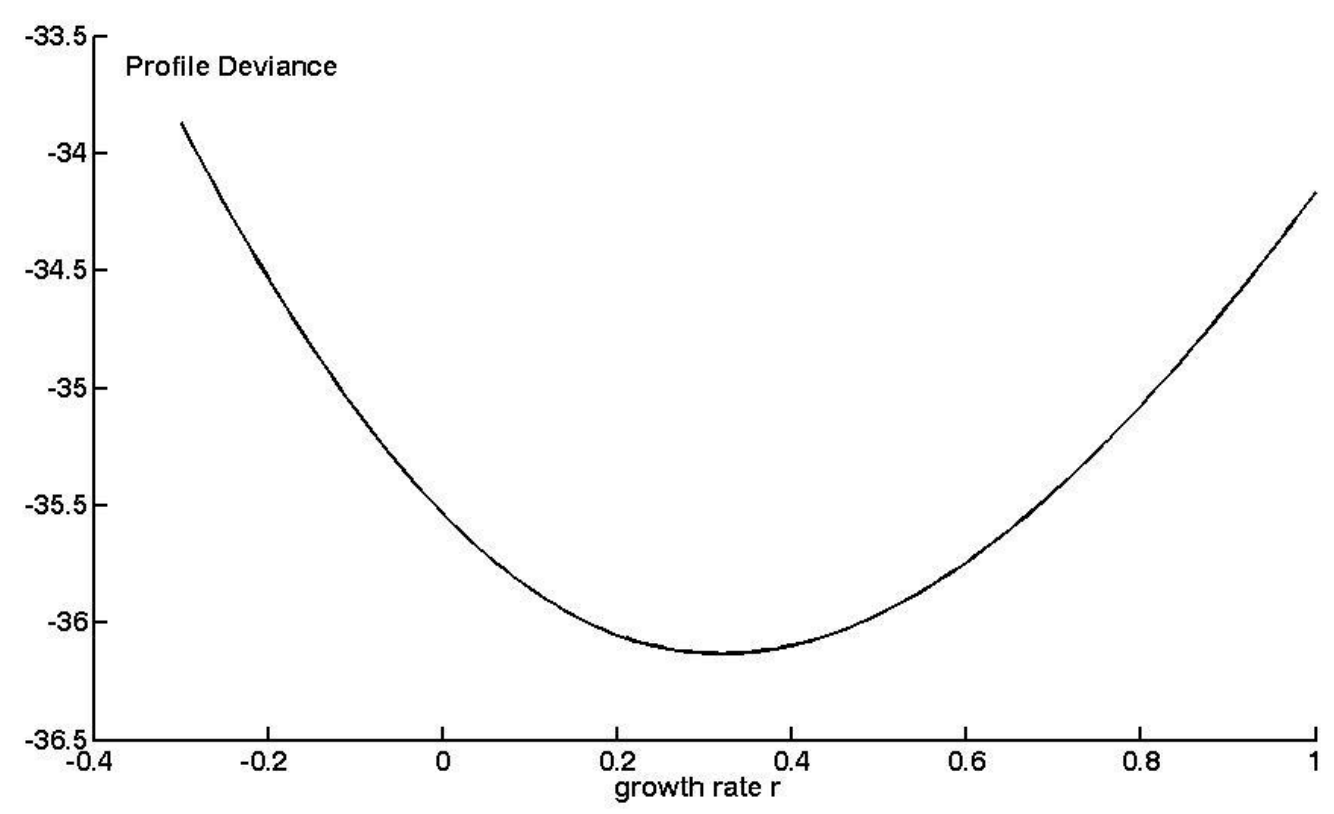

739 
Figure 5

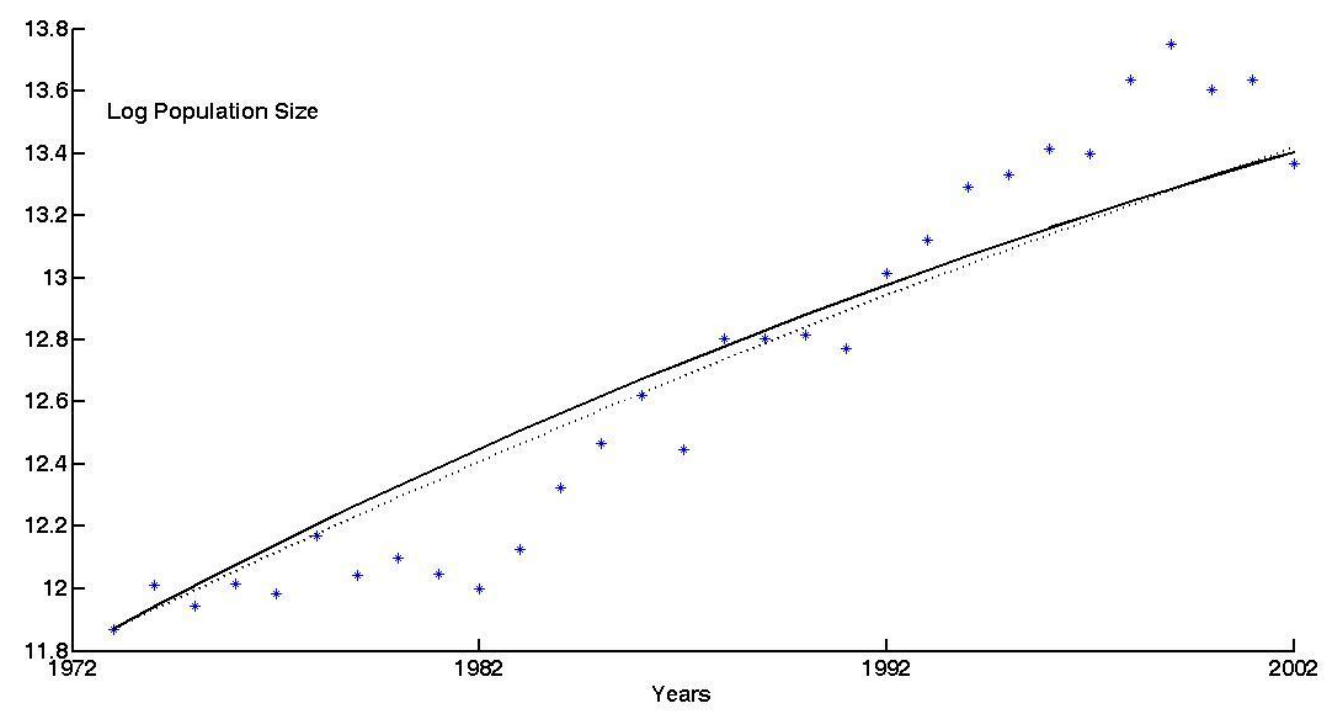

